\title{
Transvaginal resection of a rectal leiomyoma: A case report
}

\author{
NOBUHISA MATSUHASHI, TAKAO TAKAHASHI, KENGO ICHIKAWA, TOSHIYUKI TANAHASHI, \\ YOSHIYUKI SASAKI, YOSHIHIRO TANAKA, NAOKI OKUMURA, KAZUYA YAMAGUCHI, \\ SHINJI OSADA and KAZUHIRO YOSHIDA
}

Department of Surgical Oncology, Gifu University School of Medicine, Gifu 501-1194, Japan

Received December 13, 2014; Accepted July 30, 2015

DOI: $10.3892 / 01.2015 .3816$

\begin{abstract}
The present study reports the case of a patient with a rectal submucosal tumor(leiomyoma) that was resected transvaginally. A 51-year-old female presented with a rectal submucosal tumor on the anterior wall of the lower rectum, located within $3 \mathrm{~cm}$ of the anal verge. This location would normally require intersphincteric or abdominal perineal resection. However, in order to minimize the invasiveness of the treatment and reduce post-operative morbidity, transvaginal resection and laparoscopic diverting ileostomy were performed instead. With the patient under general anesthesia, the posterior vaginal mucosa was incised vertically. The tumor was then excised en bloc with the overlying rectovaginal septum and rectal submucosal tumor. A primary repair of the defect and a diverting stoma were performed. The procedure did not present any complications, and the patient was discharged on day 10 post-surgery. The diverting stoma was closed 3 months later, and the sphincter function of the patient following surgery was monitored by manometry. The results of the manometric tests indicated that the patient did not suffer from fecal incontinence. In addition, the patient did not experience anal dysfunction or discomfort following the surgical procedure.
\end{abstract}

\section{Introduction}

Submucosal tumors (SMTs) of the rectum are rare, and account for $7-8 \%$ of all leiomyomas arising in the gastrointestinal tract (1). In Japan, SMTs of the rectum are particularly rare, accounting for $\sim 1$ in every 2,000-3,000 cases of SMTs $(2,3)$. Although gastrointestinal leiomyoma is commonly detected as a SMT, definitive preoperative diagnosis is often difficult as the majority of SMTs do not cause symptoms and are identified incidentally during endoscopic or radiological examinations. The overlying mucosa typically appears smooth and normal on endoscopy (4). Therefore, the suggested diagnostic technique

Correspondence to: Professor Kazuhiro Yoshida, Department of Surgical Oncology, Gifu University School of Medicine, 1-1 Yanagido, Gifu 501-1194, Japan

E-mail:kyoshida@gifu-u.ac.jp

Key words: transvaginal resection, rectal submucosal tumor is immunohistochemical analysis (5); leiomyomas typically exhibit positivity for desmin and $\alpha$-smooth muscle actin and negativity for C-kit, CD4 and S-100. The standard surgical approaches for anorectal SMTs include transanal, transcoccygeal, transsphincteric or intersphincteric resection (ISR) for small tumors, and anterior or abdominoperineal resection for large tumors (1). However, anterior and abdominoperineal resection are associated with high mortality and morbidity (5). Recently, laparoscopic surgery, which is a minimally invasive technique, has been found to be useful for the treatment of small rectal tumors. It is associated with increased feasibility and safety with regard to the short term outcome of patients when compared with the same type of open surgery; for example, low anterior resection (6). The present study describes the case of a patient with a rectal leiomyoma who was subjected to a transvaginal resection to minimize the invasiveness of the surgery.

\section{Case report}

In February 2013, a 51-year-old female with no subjective symptoms presented to the Department of Obstetrics and Gynecology of Gifu University (Gifu, Japan) with a mass that was detected during a health screening. In January 2013, the patient underwent transvaginal ultrasonography at Mabuchi Ladies Hospital (Gifu, Japan), which demonstrated a protrusion in the anterior rectal wall with no mucosal abnormalities. The patient was then referred to the Department of Obstetrics and Gynecology of Gifu University in May 2013 for diagnosis and treatment recommendations.

Computed tomography examination detected the presence of a tumor of unclear origin that was $\sim 45 \mathrm{~mm}$ in diameter, and located between the rectum and vagina (Fig. 1). Magnetic resonance imaging revealed that the $45-\mathrm{mm}$ tumor was encapsulated by the rectal muscle, suggesting a rectal SMT, which was suspected to be a gastrointestinal stromal tumor (GIST) (Figs. 2 and 3). Colonoscopy examination identified a rectal SMT on the anterior wall of the rectum, located $3 \mathrm{~cm}$ from the anal verge (Fig. 4). On endoscopic ultrasound examination, the tumor appeared to originate from the third layer of the rectum (Fig. 5). A tumor in this region is traditionally approached with laparoscopic anterior rectal resection or ISR, which was considered. However, these approaches were not followed due to the risks associated with these procedures in terms of invasiveness and potential anal dysfunction. 


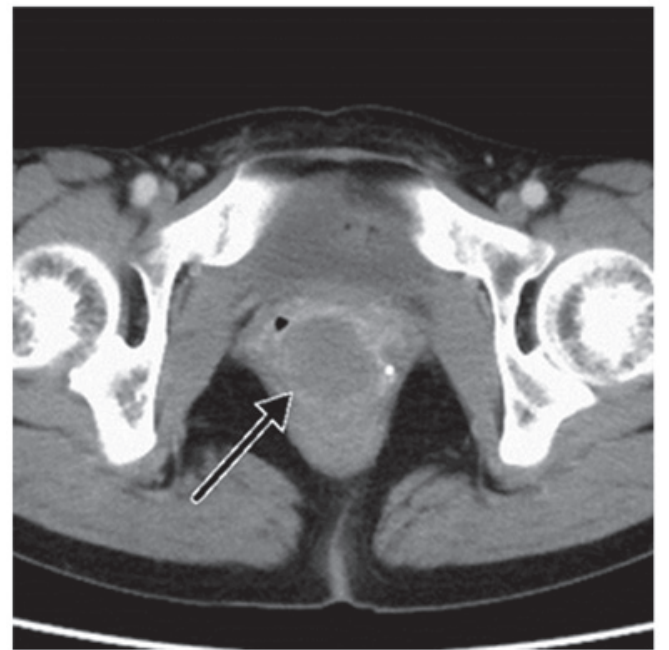

Figure 1. Computed tomography of the pelvis revealing a $45-\mathrm{mm}$ tumor (black arrow) of unclear origin located between the vagina and rectum.

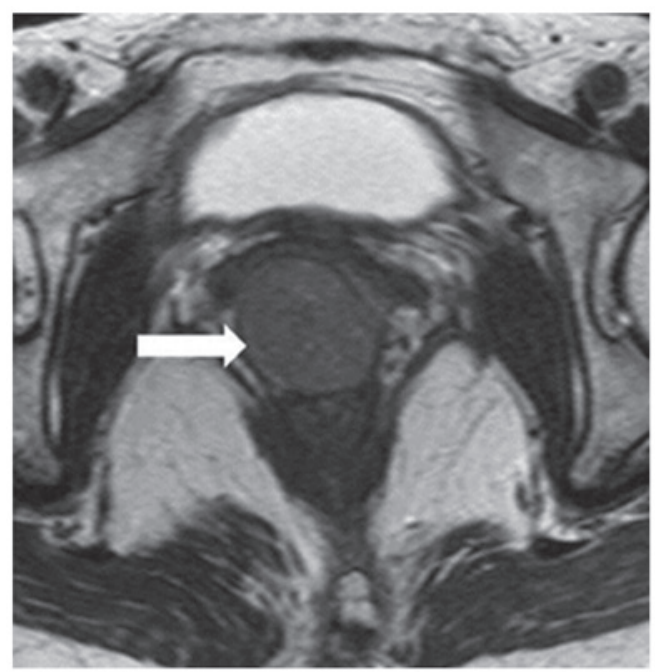

Figure 2. Magnetic resonance imaging sagittal scan of the pelvis clearly delineating the layers of the rectum and vagina, identifying the origin of the 45-mm tumor within the rectal wall (white arrow).

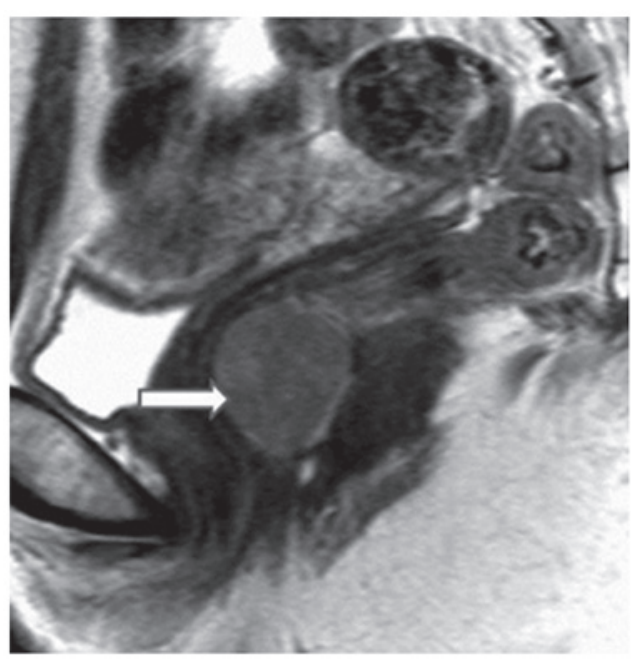

Figure 3. Magnetic resonance imaging coronal scan of the pelvis clearly delineating the layers of the rectum and vagina, identifying the origin of the $45-\mathrm{mm}$ tumor within the rectal wall (white arrow).

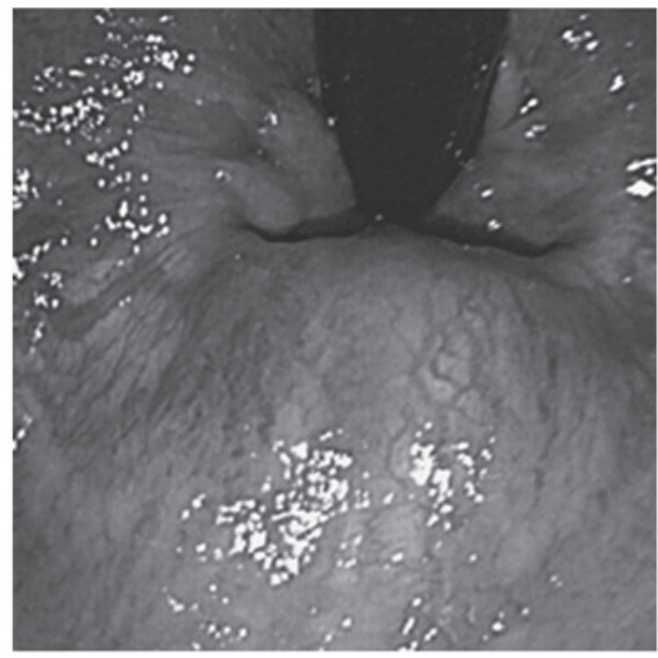

Figure 4. Colonoendoscopy image revealing a submucosal rectal tumor at the anterior wall of the nearby anal verge.

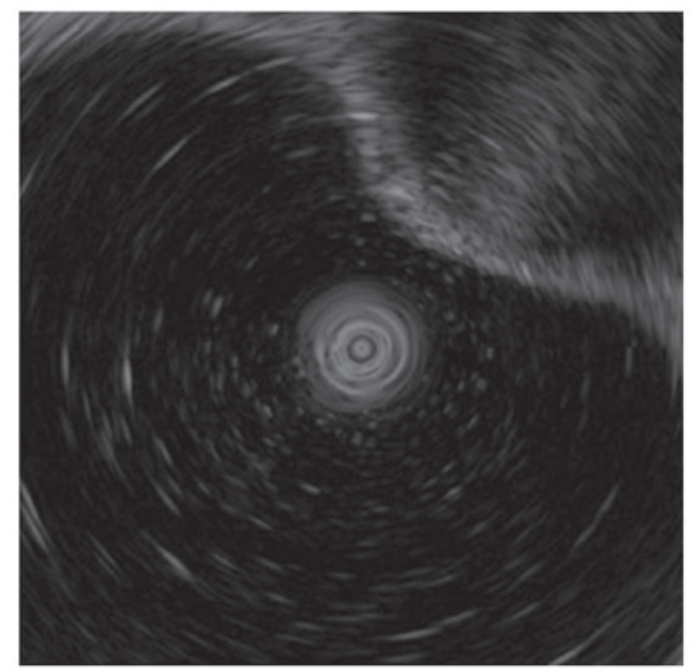

Figure 5. Colonoendoscopic ultrasonic view of the tumor demonstrating the presence of a non-invasive tumor with a homogenous echo located in the third layer of the rectum.

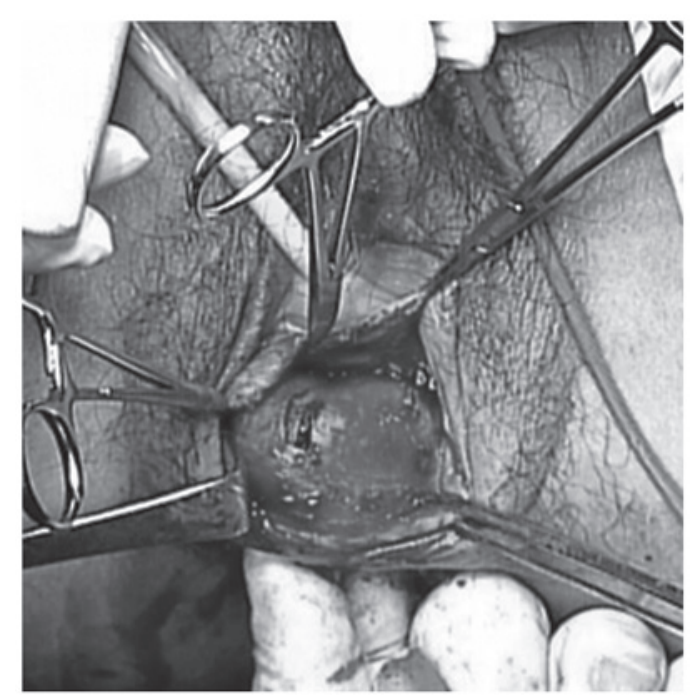

Figure 6. Transvaginal intraoperative view displaying the vaginal mucosa, which had been vertically incised from the vaginal lateral side. 


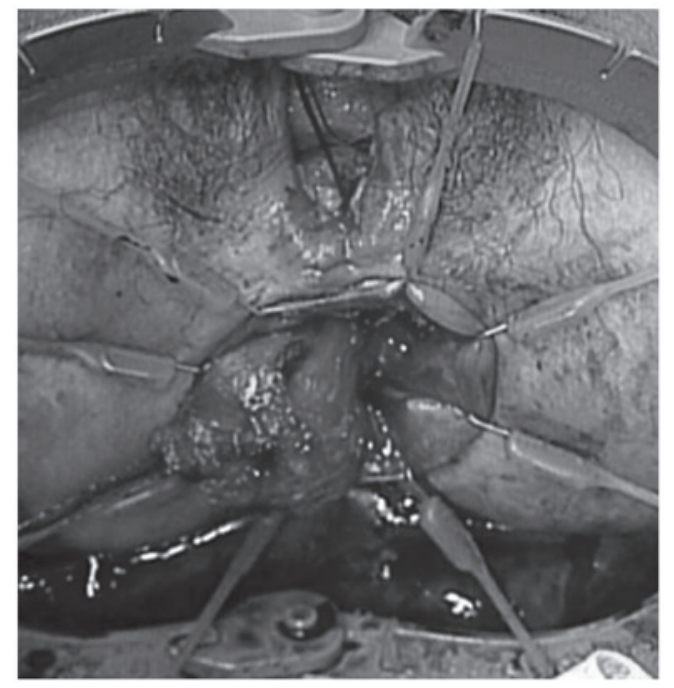

Figure 7. Transvaginal intraoperative view, from anal-to-canal side.

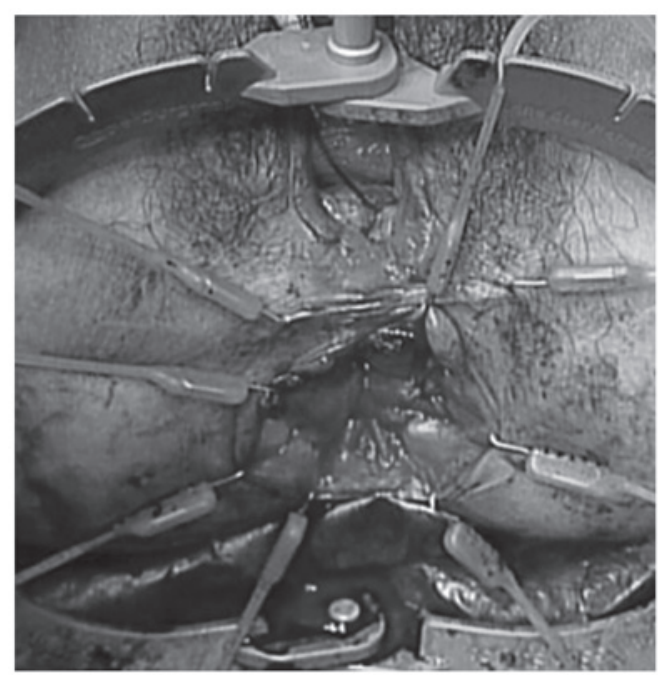

Figure 8 . Horizontally sutured rectal muscle and mucosa, with vertical closure of the vaginal layers.

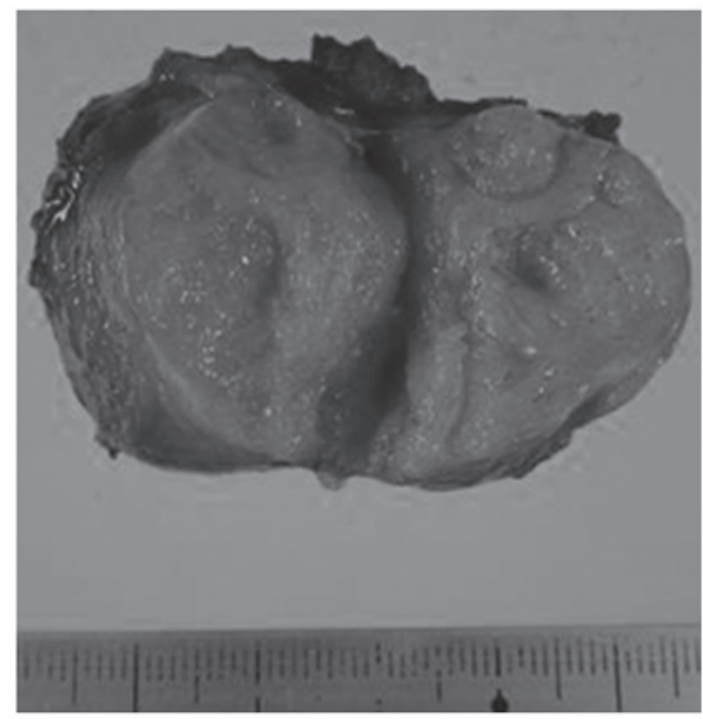

Figure 9. Macroscopic view of the resected tumor, which was encased between the rectal mucosa and submucosa.
Therefore, the patient was subjected to general anesthesia and placed in the lithotomy position. The vaginal mucosa was then incised vertically, exposing the rectovaginal septum overlying the tumor. The tumor was mobilized, and the capsule surrounding the tumor was kept intact. To prevent the development of potential complications, such as post-operative rectovaginal fistulae, the rectal muscle and mucosa were sutured horizontally, and the vaginal layers were closed vertically (Figs. 6-8). In addition, a laparoscopic diverting ileostomy via 2 ports was performed. The total surgical duration was $120 \mathrm{~min}$, and the estimated blood loss was $120 \mathrm{ml}$. Pathological examination of the tumor confirmed the diagnosis of a leiomyoma with clear surgical margins (Fig. 9). Since the recovery of the patient did not present any complications, the patient was discharged on day 10 post-surgery, and the diverting ileostomy was closed 3 months later. Currently, the Department of Surgical Oncology of Gifu University is using manometry to examine the post-operative sphincter status of all the patients who underwent ISR and ultra-low anterior resection at their premises. Fecal incontinence in these post-operative patients is evaluated with three-dimensional vector manometry to determine the median of the maximum resting and squeeze pressures in the anal sphincter. In addition, the modified Fecal Incontinence Quality of Life (mFIQL) scale and Wexner score (7) are used to assess the patients. The defecatory function is assessed in terms of frequency of bowel movements and continence. The median maximum resting pressure measured for the patient of the current report was $25.0 \mathrm{mmHg}$ (normal range, 50.3-92.2 $\mathrm{mmHg}$ ), and the maximum squeeze pressure was $118.6 \mathrm{mmHg}$ (normal range, 100.3-240.8 $\mathrm{mmHg}$ ). The $\mathrm{mFIQL}$ and Wexner scores of the patient at 3 months following the closure of the stoma were each 0 points, indicating the absence of fecal incontinence. Furthermore, thus far, the patient has not experienced any anal dysfunction or discomfort post-operatively. Written informed consent was obtained from the patient for the publication of the present case report and any accompanying images.

\section{Discussion}

Rectal surgery, and particularly surgery on the lower rectum, is associated with more complications than other surgical procedures performed on more proximal portions of the gastrointestinal tract. These complications include anastomotic leakage, bleeding, impaired anal sphincter function and fecal incontinence. The resection of tumors of the rectum is difficult and invasive due to the location of the rectum on the pelvic floor (8). Surgical procedures on the lower rectum normally require dissection down to the levator ani muscle, which may result in anal dysfunction or discomfort (1). Therefore, a less invasive procedure is desirable $(9,10)$.

SMTs of the rectum are relatively rare, and include GISTs, carcinoids, leiomyomas, leiomyosarcomas, malignant lymphomas, neurinomas and lipomas. Sasaki et al (11) reported the recurrence of relapse in 5 out of 76 patients with SMTs, and Kusminsky and Bailey (12) reported a recurrence rate of $31 \%$ in affected patients.

The main problem associated with a rectal leiomyoma is the histological diagnosis, since the pathological image does not usually correspond to the biological malignancy (11). 
In the present study, the diagnosis of a suspected GIST was confirmed by endoscopic ultrasound examination.

Although rectal GISTs and SMTs exhibit malignant potential, patients with rectal GISTs present with equivalent survival rates, regardless of whether a local or complete bowel resection is performed (11). This is possibly due to the preference of this type of tumor for metastasizing hematogenously rather than via the lymphatic system. Similarly, regional lymphadenectomy confers no additional benefit. Vorobyov et al (13) described a vaginal approach for the removal of a leiomyoma that was located in the rectovaginal wall in 1 out of 36 patients treated for rectal tumors. The study also reported the following rates of surgery performed by different methods: Electroexcision, $33.3 \%$; transanal excision, $27.8 \%$; removal of the rectum via the pararectal approach, 16.7\%; abdominal resection, $13.9 \%$; extirpation of the rectum, $5.6 \%$; and trans-vaginal excision, $2.7 \%$. In addition, the study noted that complications in the early post-operative period were observed in $33.5 \%$ of patients. Visser et al (14) also reported that these procedures were associated with a high complication rate, particularly with regards to fistulae, which occurred in $21 \%$ of patients.

Transvaginal local excision for rectal carcinoma was also performed by $\mathrm{Fu}$ et al (8) in a series of 18 patients with $\mathrm{T} 1$ and T2 rectal cancer. For this approach, the average distance from the dentate line best fit is $\sim 4 \mathrm{~cm}$. The potential complication of rectovaginal fistula was reported in 1 out of the 18 patients, and was treated conservatively. The use of the transvaginal approach was also reported by Hellan and Maker (15) for the excision of an anterior anorectal $5 \times 5 \times 8-\mathrm{cm}$ GIST, and by Hara et al (16) for the excision of an anterior anorectal GIST that was $\sim 5 \mathrm{~cm}$ in size. None of these studies described any complications post-surgery or the requirement for a diverting ileostomy. Furthermore, no recurrence was observed.

Preventing recurrence and enhancing the quality of life of patients with small GISTs or leiomyoma are of importance due to the low malignancy associated with these conditions, as resection may not be necessary if such tumors are asymptomatic. Therefore, in order to prevent the potential creation of a rectovaginal fistula in the present patient during the restoration of the injured part of the rectum, a diverting ileostomy was created via laparoscopic surgery. The anal function of the patient following surgery was examined throughout the post-operative course, and was observed to be excellent, as indicated by the mFIQL and Wexner scores $(7,17)$. To the best of our knowledge, the present study is the first case reporting the use of a transvaginal resection for the excision of a lower rectal tumor. This transvaginal approach for the excision of an anterior anorectal SMT in the lower rectum provides a reasonable alternative to traditional surgical procedures, which usually are affected by post-operative complications.
In conclusion, the present study reports the case of a patient with an SMT of the lower rectum that was successfully resected transvaginally. This approach is less invasive than conventional methods and offers a low risk of anal dysfunction. Therefore, the transvaginal resection of rectal tumors should be considered more often.

\section{References}

1. Hatch KF, Blanchard DK, Hatch GF III, Wertheimer-Hatch L, Davis GB, Foster RS Jr and Skandalakis JE: Tumors of the rectum and anal canal. World J Surg 24: 437-443, 2000

2. Anderson PA, Dockerty MB and Buie LA: Myomatous tumors of the rectum (leiomyomas and myosarcomas). Surgery 28: 642-650, 1950.

3. Serra J, Ruiz M, Lloveras B, Guillaumes S, Garriga J and Trias R: Surgical outlook regarding leiomyoma of the rectum. Report of three cases. Dis Colon Rectum 32: 884-887, 1989.

4. Yasuda K, Nakajima M, Yoshida S, Kiyota K and Kawai K: The diagnosis of submucosal tumors of the stomach by endoscopic ultrasonography. Gastrointest Endosc 35: 10-15, 1989.

5. Miettinen M, Furlong M, Sarlomo-Rikala M, Burke A, Sobin LH and Lasota J: Gastrointestinal stromal tumors, intramural leiomyomas, and leiomyosarcomas in the rectum and anus: A clinicopathologic, immunohistochemical, and molecular genetic study of 144 cases. Am J Surg Pathol 25: 1121-1133, 2001.

6. Fujimoto Y, Akiyoshi T, Kuroyanagi H. Konishi T, Ueno M, Oya $\mathrm{M}$ and Yamaguchi T: Safety and feasibility of laparoscopic intersphincteric resection for very lower rectal cancer. J Gastrointest Surg 14: 645-650, 2010.

7. Hashimoto H, Shiokawa H, Funahashi K, Saito N, Sawada T, Shirouzu K, Yamada K, Sugihara K, Watanabe T, Sugita A, et al: Development and validation of a modified fecal incontinence quality of life scale for Japanese patients after inter-sphincteric resection for very low rectal cancer. J Gastroenterol 45: 928-935, 2010.

8. Fu T, Liu B, Zhang S, Wang D and Zhang L: Trans-vaginal local excision of rectal carcinoma. Curr Surg 60: 538-542, 2003.

9. Rullier E, Laurent C, Carles J, Saric J, Michel P and Parneix M: Local recurrence of low rectal cancer after abdominoperineal and anterior resection. Br J Surg 84: 525-528, 1997.

10. Wibe A, Syse A, Andersen E, Tretli S, Myrvold HE and Søreide O; Norwegian Rectal Cancer Group: Oncological outcomes after total mesorectal excision for cure for cancer of the lower rectum: Anterior vs. abdominoperineal resection. Dis Colon Rectum 47: 48-58, 2004.

11. Sasaki K, Gotoh Y, Nakayama Y, Hayasaka H, Ishiyama Y and Miyashita H: Leiomyoma of the rectum. Int Surg 70: 149-152, 1985.

12. Kusminsky RE and Bailey W: Leiomyomas of the rectum and anal canal: Report of six cases and review of the literature. Dis Colon Rectum 20: 580-599, 1977.

13. Vorobyov GI, Odaryuk TS, Kapuller LL, Shelygin YA and Kornyak BS: Surgical treatment of benign, myomatous rectal tumors. Dis Colon Rectum 35: 328-331, 1992.

14. Visser BC, Varma MG and Welton ML: Local therapy for rectal cancer. Surg Oncol 10: 651-652, 2001.

15. Hellan M and Maker VK: Trans-vaginal excision of a large rectal stromal tumor: An alternative. Am J Surg 191: 121-123, 2006.

16. Hara M, Takayama S, Arakawa A, Sato M, Nagasaki T and Takeyama H: Trans-vaginal resection of a rectal gastrointestinal stromal tumor. Surg Today 42: 909-912, 2012.

17. Jorge JM, Wexner SD, Morgado PJ Jr, James K, Nogueras JJ and Jagelman DG: Optimization of sphincter function after the ileoanal reservoir procedure. A prospective, randomized trial. Dis Colon Rectum 37: 419-423, 1994. 